\title{
Bender-type Tooth-Movement Transducer
}

$\begin{array}{lcc}\text { Member } & \text { Hisao Oka } & \text { (Okayama University) } \\ \text { Member } & \text { Yoshikazu Shimizu } & \text { (Okayama University) } \\ \text { Non-member } & \text { Keiji Saratani } & \text { (Osaka Dental University) } \\ \text { Non-member } & \text { Sheng-gen Shi } & \text { (Fourth Military Medical University) } \\ \text { Non-member } & \text { Takayoshi Kawazoe } & \text { (Osaka Dental University) }\end{array}$

Summary

A tooth-movement transducer using bender-type piezoelectric ceramics was developed for the measurement probe of the Tooth Mobility tester. It is small, light and suitable for oral examination and it is possible to evaluate the tooth mobility of a molar in the direction of the tooth axis. The transducer utilizes a set of long and rectangular ceramic beam. It includes two supporting points and it is possible to adjust the resonance frequency by moving two points. This frequency is applied as the measuring frequency. The basic characteristics of the transducer and clinical applicability of the measurement probe are confirmed using an artificial tooth model and human tooth.

Keywords: bender-type, tooth mobility, mechanical mobility, piezoelectric ceramics, tooth movement

\section{INTRODUCTION}

Tooth mobility examination is important in planning dental treatment and in evaluating its results. A manual examination of tooth mobility is regularly carried out in dental clinics with the fingers or dental hand-held instruments. This, however requires skill and experience [1]. The PERIOTEST has been designed to measure tooth mobility objectively [2]. An electronically controlled rod percusses the tooth and it is decelerated when it impinges on the tooth. After hitting the tooth, the rod recoils. This contact time represents the measuring parameter proper. The force of the impact rod on the tooth, however, is sometimes strong and can cause pain. The authors have previously reported on an automatic diagnosis system for tooth mobility in clinical use, by applying a small random vibration $(30-1000 \mathrm{~Hz})$ on the tooth [3]. However it is fairly large because of the requirement for a personal computer for a data sampling and analysis.

In a previous study, the authors have already developed a "Tooth Mobility (T-M) Tester" for evaluating the tooth mobility objectively and quantitatively [4]. The developed tester is composed of a measurement probe and system amplifier. A single-frequency vibration is applied to the tooth surface, an acceleration response is measured, and then the mechanical mobility of the periodontium is calculated. The tooth-movement transducer of the probe utilizes a set of bimorph piezoelectric ceramic disks. It is therefore small, light, and suitable for oral examination. It is, however, difficult to measure the tooth mobility of the molars in the direction of the tooth axis. Then it has been expected that a smaller probe would be developed. It is, however, difficult to make a modified, smaller probe and to adjust the measuring frequency because of the structure of the previous probe.

In this study, the authors have developed a new measurement probe using a transducer composed of bender-type piezoelectric ceramics. The transducer utilizes a long and rectangular ceramic plate which is smaller than the one used previously [5]. This paper deals with the basic characteristics of the newlydeveloped transducer and the clinical applicability of the measurement probe.

\section{TOOTH MOBILITY TESTER}

$<2.1>\mathrm{T}$-M Tester In the developed T-M tester, a single sinusoidal vibrational force is applied to the tooth surface and an acceleration response is detected using the transducer of the measurement probe. A block diagram of the tester is shown in Figure 1 [4]. A The acceleration signal, which is amplified by means of a charge amplifier, is converted to a root-meansquare value using a RMS/DC circuit. Unnecessary movements from probe handling are eliminated using a $100 \mathrm{~Hz}$ high-pass 
filter.

The contact pressure of the probe on the tooth surface in acceleration measurement should be maintained because the physical properties and functions of the periodontal ligament depend on both axial and lateral loads [1]. Four strain gauges, which are glued to the ceramic surface of the transducer detect the contact pressure. When the detected pressure matches 50 $\pm 5 \mathrm{gf}$ (gram-force) and maintains it for $0.1 \mathrm{sec}$, the acceleration $\mathrm{r}-\mathrm{m}$-s signal is automatically sampled. When the pressure is below 45 or over $55 \mathrm{gf}$, an alarm is sounded and the operator should adjust the contact pressure of the probe on the teeth. The sampled data are transformed to a series of pulses and are counted for $0.5 \mathrm{sec}$. After this sampling and counting of data is repeated ten times, an alarm signalling completion is sounded, and the total number of pulses is presented on a digital panel counter. Its reading corresponds to the magnitude of mechanical mobility, i.e. the "Mobility Index $(M I)$ " score, as described later. When periodontal pathology advances and tooth movability becomes greater, the $M I$ score increases, corresponding to its mechanical mobility.

$<2.2>$ Mobility Index The biomechanical mobility $\lambda$ (a reciprocal of mechanical impedance) of the periodontium is defined as $\lambda=V / F=A / 2 \pi f F$ ( $F$ : force, $A$ : acceleration, $V$ : velocity, $f$ : vibrating frequency), when a single sinusoidal vibration is applied to the tooth surface and an acceleration is detected at the driving point. The mechanical mobility of the periodontium expresses the tooth movability, which was proposed by Noyes et. al. [6]. The mobility index of tooth movability is defined as $M I=\mathrm{K} A=\mathrm{K} 2 \pi f F \lambda(\mathrm{K}$ : proportional constant), but it is a dimensionless quantity. When $F$ and $f$ are constant, the $M I$ score is proportional to $A$ and $\lambda$. Because the mechanical mobility of the periodontium is typical of tooth movability, it is proper to evaluate the tooth mobility using the $M I$ score

\section{BENDER-TYPE MEASUREMENT PROBE}

<3.1> Design of Measurement Probe Figure 2 shows a measurement probe using bender-type piezoelectric ceramics transducer. The measurement probe, which was developed for the T-M tester previously, utilized a set of piezoelectric ceramic disks [7]. The ceramic disks were $25.5 \mathrm{~mm}$ in diameter and $100 \mu \mathrm{m}$ thick, with the fringe fixed with a holding ring. The shim between the two ceramics is made of $\mathrm{Be}-\mathrm{Cu}$ and is 100 $\mu \mathrm{m}$ thick. When the resonance frequency of the transducer is used as the actuating frequency for the transducer, the acceleration response is larger and more sensitive [4]. The disktype ceramics of smaller size, however, require a higher resonance frequency. In order to decrease the resonance frequency within a range from $300-500 \mathrm{~Hz}$ [7], it is necessary to reduce the thickness of the ceramics and shim of the transducer or to increase the diameter of the transducer. Unfortunately, the former produces a fragile transducer and the latter does not satisfy the plan of miniaturization.

In this study, the tooth movement transducer includes a set of bimorph bender-type ceramics. The PZT ceramics are long, rectangular plates of $60(\mathrm{~L}) \times 5(\mathrm{~W}) \mathrm{mm}$ and $180 \mu \mathrm{m}$ thickness. The shim between the two ceramics is made of $\mathrm{Be}-\mathrm{Cu}$ and is

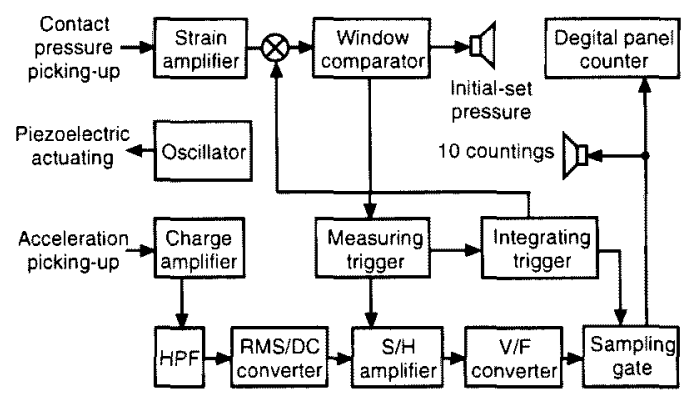

Fig. 1 Block diagram of T-M tester.

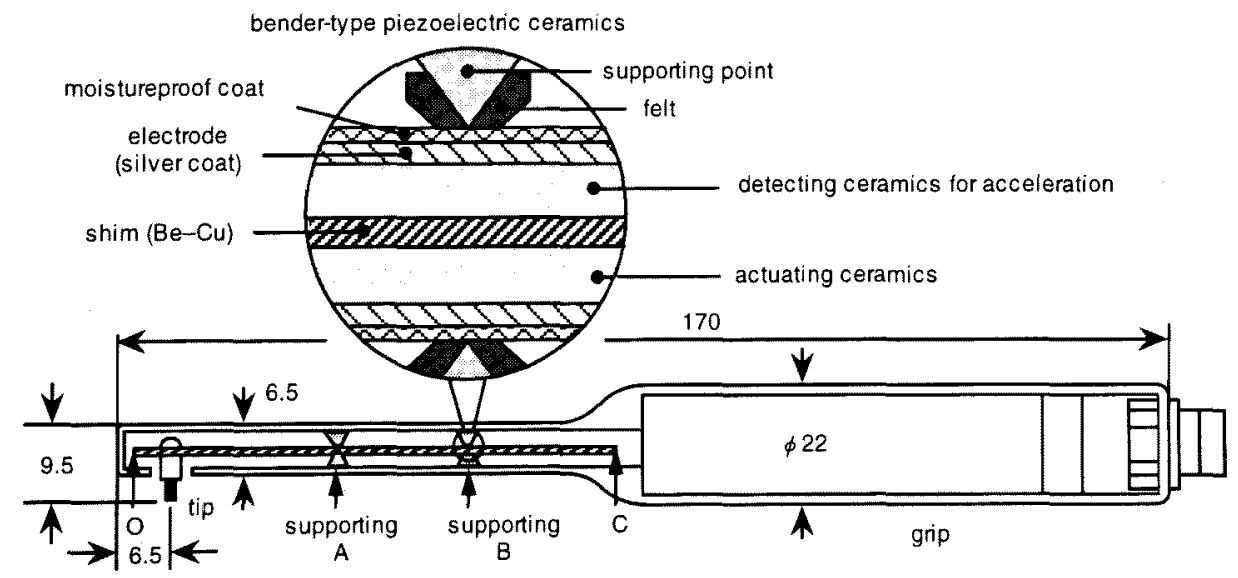

Fig. 2 Bender-type piezoelectric ceramics. 
63(L) $\times 5(\mathrm{~W}) \mathrm{mm}$ and $100 \mu \mathrm{m}$ thickness. The two ceramics polarized in reverse directions are deposited onto both sides of the shim. The piezoelectric strain constant of ceramic is $-360 \mathrm{x}$ $10^{-12} \mathrm{~m} / \mathrm{V}$ in $d_{31}$. The surface of the ceramic is covered with a moistureproof coat. One of the bimorph ceramics is used for actuating and the other for detecting acceleration. Because the newly-developed transducer is short, narrow and about $500 \mu \mathrm{m}$ in total thickness, it is more suitable as a tooth-movement transducer used in mouth. A cotton felt of about $2 \mathrm{~mm}$ thickness held at a supporting point suppresses the high metallic $Q$ of the shim and avoids any slipping out caused by a shock.

A photograph of the newly developed measurement probe using bender-type piezoelectric ceramics is shown in Figure 3. The probe has an overall length of $170 \mathrm{~mm}$ and is about $100 \mathrm{~g}$ in weight. The pointed end of the probe is thin and small-sized at $6.5 \mathrm{~mm}$ in thickness and $11.5 \mathrm{~mm}$ in width, so as to be easily inserted into the mouth. It then becomes easier to measure the tooth mobility of the periodontium from every angle in mouth. Inside the probe's grip, which is $22 \mathrm{~mm}$ in diameter, is a space for an electric driver and preamplifier, making the whole T-M tester portable for clinical use.

<3.2> Adjustment of Resonance Frequency When it is assumed that this rectangular transducer behaves as a cantilever beam (clamped and free end), the resonance frequency is

$$
f=\frac{\alpha^{2}}{4 \sqrt{3} \pi} \cdot \frac{T}{L^{2}} \sqrt{\frac{Y}{\rho}}
$$

where $T$ is the thickness of beam, $L$ is the length of the beam, $Y$ is the Young's modulus, $\rho$ is the density of the beam and $\alpha$ is the boundary condition constant [8]. When the material and the $\alpha$ of the beam are determined, the resonance frequency depends on the thickness and length of the beam. It is apparent that the resonance frequency decreases by lengthening instead of thinning the beam according to Eq. (1). Moreover, it is possible to make the beam narrower because the resonance frequency is independent of the beam width. In this way, the bender-type transducer achieves mechanical strength and allows for a miniaturization of the measurement probe.

The transducer beam includes two supporting points. By changing the distances among $\mathrm{OA}, \mathrm{AB}$ and $\mathrm{BC}$, it is possible to adjust the resonance frequency. The acceleration spectrum of the bender-type transducer is shown in Figure 4. The solid line is the spectrum in the case of $O A=10, A B=20$, and $B C=33$ $\mathrm{mm}$. The gray line is in the case of $\mathrm{OA}=14, \mathrm{AB}=20$, and $\mathrm{BC}=29$ $\mathrm{mm}$. The distance between the $A$ and $B$ supporting points is kept at $20 \mathrm{~mm}$ and the applied pressure to the beam at the point is $50 \mathrm{gf}$. When the supporting points $\mathrm{A}$ and $\mathrm{B}$ move parallel to one another in the direction of the point $C$, the spectrum includes two sharp resonances, $\mathrm{f}_{\mathrm{BC}}$ and $\mathrm{f}_{\mathrm{OA}}$. As described later, these resonances are related to the length of $\mathrm{OA}$ and $\mathrm{BC}$, respectively. Namely, the shorter $\mathrm{OA}$ distance is related to the high-resonance frequency $\mathrm{f}_{\mathrm{OA}}$, and the longer $\mathrm{BC}$ distance is related to the low-resonance frequency $f_{B C}$. The figure shows that as $O A$ becomes longer, the $f_{O A}$ becomes lower, and as $B C$ becomes shorter $f_{B C}$ becomes higher.

$<3.3>$ Characteristics of the transducer Figure 5 shows a mechanical mobility spectrum for the measurement probe with the newly-developed bender-type piezoelectric ceramics where the supporting points are kept at $\mathrm{OA}=23, \mathrm{AB}=14, \mathrm{BC}=26$ $\mathrm{mm}$. In the case of nocontact with any object, the probe involves four resonances in the spectrum below $5 \mathrm{kHz}$, shown in the figure (a). When a part of the transducer OA touches some stiff object such as resin, $\mathrm{f}_{O A}(=300 \mathrm{~Hz})$ and $\mathrm{f}_{O A},(=2137 \mathrm{~Hz})$ shrink but $f_{B C}(=500 \mathrm{~Hz})$ and $f_{B C}(=3275 \mathrm{~Hz})$ do not change, shown in the figure (b). Conversely, it is also confirmed that $f_{B C}$ and $f_{B C}$, shrink, and $f_{O A}$ and $f_{O A}$ do not change, when a part of the transducer $B C$ touches something. From the above discussion it becomes clear that a vibrating condition related to $O A$ determines the resonance frequency $f_{O A}$ and conversely $B C$ determines $f_{B C}$. It is therefore confirmed that each vibration related to $\mathrm{OA}$ and $\mathrm{BC}$ is independent. The resonance frequency in Eq. (1) gives a fundamental frequency of the cantilever beam with the end clamped and the other end free. The resonance frequency for the second mode of vibration with an additional node is 6.267 times the fundamental frequency. The resonance frequency for the third mode with two nodes is 17.55 times the

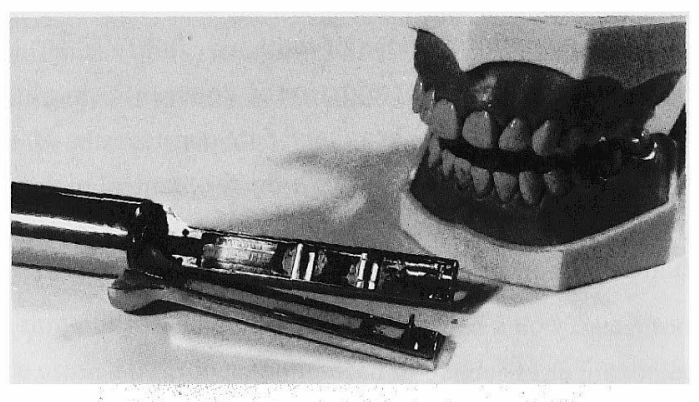

Fig. 3 Bender-type measurement probe.

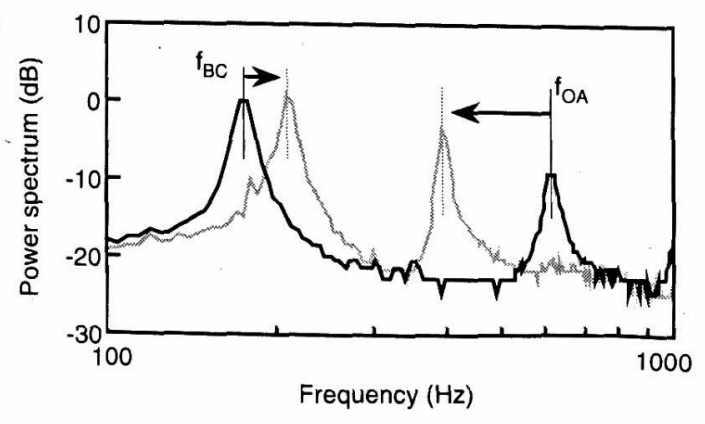

Fig. 4 Two sharp resonances of the transducer. 
fundamental frequency [9]. $f_{O A}$ is 7.12 times the fundamental frequency $f_{O A}$ and $f_{B C}$ is 6.55 times the frequency $f_{B C}$. It is therefore suggested that $f_{O A}$ and $f_{B C}$ are the frequencies for the second mode of vibration, respectively.

\section{MI SCORES OF TOOTH MODEL AND HUMAN INCISOR}

Figure 6 shows a mechanical mobility spectrum for the probe below $500 \mathrm{~Hz}$. In this figure, a solid line represents the spectrum for cases in which the measuring tip does not come into contact with any other object. The spectrum involves two sharp resonances, a low resonance frequency $f_{O A}$ of $300 \mathrm{~Hz}$ and a high resonance frequency $f_{B C}$ of $500 \mathrm{~Hz}$ (see Fig.5). The frequency $f_{O A}$ is applied as the measuring frequency for the measurement probe. As it is within the frequency range of 300 $500 \mathrm{~Hz}$ which is suitable for human teeth [7], it is sufficient as an artificial model. The other lines indicate spectra which are measured using an artificial tooth model shown in Figure 7.

The tooth model includes four teeth whose periodontium were diagnosed with clinical tooth mobilities of M0, M1, M2 and $M 3$, respectively. M0 indicates no perceptible movement and a healthy and clinically firm tooth. M3 indicates a degree of mobility indicative of a hopeless prognosis [10]. The artificial periodontal ligaments are made of silicone impression material of thickness 0 (M0), 0.28 (M1), 0.56 (M2), and $0.84 \mathrm{~mm}$ (M3). Because it is supposed that the width of the periodontal ligament is one of the causes of pathological tooth mobility [7]. The dotted, gray-dotted, broken and gray-broken lines in Fig. 6 represent four spectra (encircled) for cases in which the tip comes into contact with the M0, M1, M2 or M3 tooth at $50 \mathrm{gf}$, respectively. The M0 tooth is clinically firmer than the M 3 tooth and the greater the tooth mobility, the larger the mechanical mobility. It can be seen in the figure that the spectrum magnitudes for M0 to M3 are significantly different at $300 \mathrm{~Hz}$. It is clear that the mechanical mobility spectrum is different depending upon the tooth mobility.

Table 1 shows the average and standard deviation of tooth mobility index $M I$ scores for four teeth using the artificial tooth model. These values were obtained using the $T-M$ tester equipped with the newly-developed measurement probe using a measuring frequency of $300 \mathrm{~Hz}$. The coefficient of variation (standard deviation / mean value) in ten measurements is within $1 \%$. The results can be explained well, and the differences between $M 0, M 1, M 2$ and $M 3$ are clear from the table. A slight deviation indicates a precise measurement. Next, the tooth mobility of human maxillary left central incisor with a healthy periodontium is measured ten times. The $M I$ score is also shown in the table. The data clearly show that the T-M tester and measurement probe are applicable for clinical use.

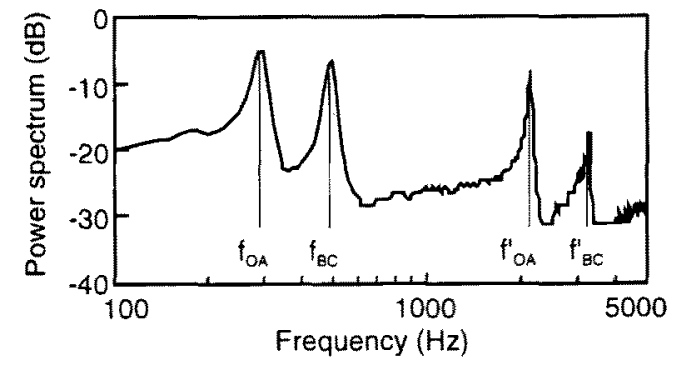

(a) four resonances of the probe

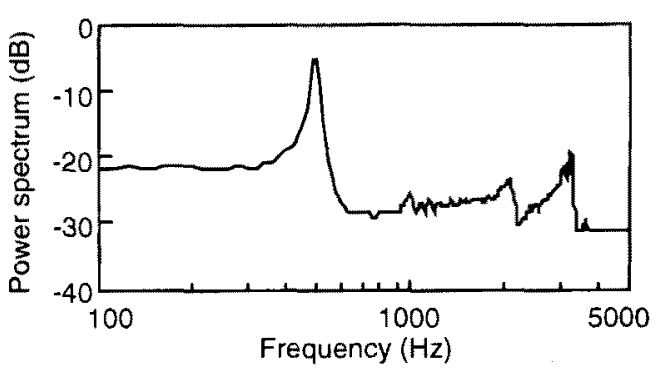

(b) shrink of $f_{O A}$ and $f_{O A}$

Fig. 5 Mechanical mobility spectra of measurement probe in the case of no/contact with any object.

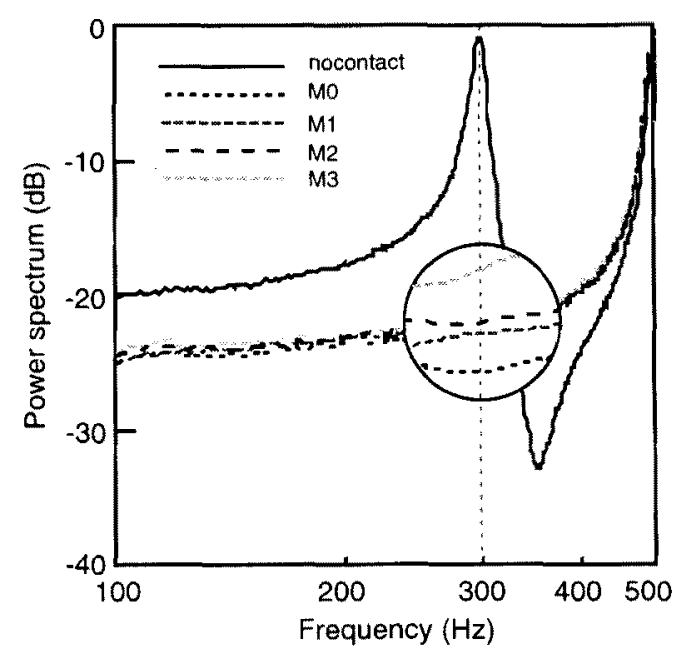

Fig. 6 Mechanical mobility spectra for the probe in the case of contact with teeth.

\section{CONCLUSIONS}

A tooth-movement transducer using bender-type piezoelectric ceramics was developed for the measurement probe of the T-M tester. The transducer is $63(\mathrm{~L}) \times 5(\mathrm{~W}) \mathrm{mm}$ and $500 \mu \mathrm{m}$ thickness. The transducer plate has two supporting points which can adjust the resonance frequency of transducer by changing the distance between two points. The measurement 


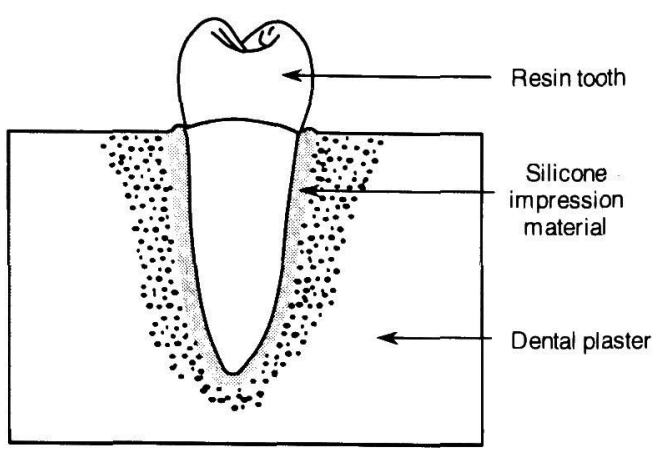

Fig. 7 Artificial tooth model.

Table 1 MI scores of tooth model and human incisor.

\begin{tabular}{|c|c|c|c|c|c|c|}
\hline \multicolumn{2}{|c|}{ teeth } & M0 & M1 & M2 & M3 & incisor \\
\hline \multirow{2}{*}{$\begin{array}{c}M I \\
\text { score }\end{array}$} & mean & 7531 & 7746 & 7828 & 8156 & 7480 \\
\cline { 2 - 7 } & SD & 25 & 25 & 12 & 19 & 26 \\
\hline
\end{tabular}

probe using this transducer is small, light and suitable for oral examination. It is possible to evaluate the tooth mobility of a molar in the direction of the tooth axis using this probe. This paper describes the basic characteristics of the transducer and the clinical applicability of this measurement probe is confirmed using an artificial tooth model and human tooth. The coefficient of variation in ten measurements is within 1 $\%$.

\section{Acknowledgments}

The authors are grateful to S.Tanida, K.Hirai and T.Mutoh of Megasera Inc. and to A.Tachibana and K.Matoba of J.Morita MFG.Corp. for their considerable assistance with the trial apparatus. The authors also would like to acknowledge the Scientific Research Fund of the Japanese Ministry of Education, Sports, Science and Culture (No.08455198) for its support. (Manuscript received March 24, 1997, revised July 7, 1997)

\section{REFERENCES}

[1] B.K.B.Berkovitz, B.J.Moxham and H.N.Newman, "The periodontal ligament in health \& disease," Mosby-Wolfe, 1995.

[2] B.d'Hoedt, D.Lucus, L.Mühlbradt F.Scholz, W.Schulte, F.Quante and A.Topkaya, "Das PeriotestverfahrenEntwicklung und klinische Prüfung," Dtsch Zahnärztl., vol.Z-40, pp.113-125, 1985.

[3] H.Oka, T.Yamamoto, K.Saratani and T.Kawazoe, "Automatic diagnosis system of tooth mobility for clinical use," Medical Progress through Technology, vol.16, pp.117-124, 1990.

[4] H.Oka, T.Irie, K.Yasuhara and T.Yamamoto, "Transducer for measuring skin stiffness," Sensors and Materials, vol.4, no.6, pp.337-349, 1993.

[5] H.Oka, Y.Shimizu, K.Saratani and T.Kawazoe, "Tooth mobility tester using a bender-type piezoelectric transducer," IEICE Trans. (in printing).

[6] D.H.Noyes and C.W.Solt, "Measurement of mechanical mobility of human incisors with sinusoidal forces," J.Biomechanics, vol.6, pp.439-442, 1973.

[7] H.Oka, K.Yasuhara K.Saratani, T.Nakanishi, M.Tatsuta, T.Kawazoe and K.Hirai, "Tooth movement sensor for clinical use," Sensors and Materiels, vol.9, no.1, pp.1-14, 1997.

[8] C.M.Harris and C.E.Crede, "Shock and vibration handbook," MaGraw-Hill Book Company, 1976.

[9] J.L.Meriam, "Mechanics part 1: statics," John Wiley \& Sons Inc., 1959.

[10] D.A.Grant, I.B.Stern and F.G.Everett, "Periodontics in the tradition of orban and gottlieb," The C.V.Mosby Company, 1979.

Hisao Oka received $\mathrm{BE}$ and $\mathrm{ME}$ degrees in Electrical

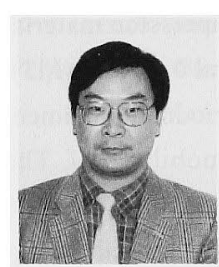
Engineering from Okayama University, Japan in 1976 and 1978, respectively, and completed a DE degree in Biomechanical Property Measurement at Osaka University in 1988. He is presently a associate professor in the Department of Electrical and Electronic Engineering, Faculty of Engineering, Okayama University. He is currently interested in measurements and applications of biomechanical properties.

Yoshikazu Shimizu received BE degree in Electrical and

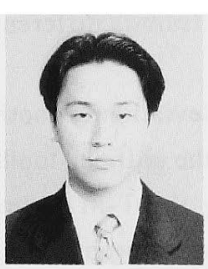
Electronic Engineering from Okayama University, Japan in 1996. He is presently a student of ME degree in the course of Electrical and Electronic in graduate school of Engineering, Okayama University. He is currently interested in measurement of tooth movement.

Keiji Saratani received his D.D.S. degree from Osaka Dental

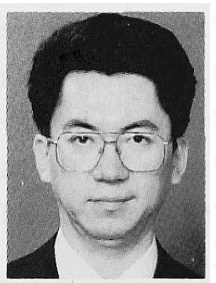
University, Osaka, Japan in 1980 and his Ph.D there in 1984 . He is presently a lecturer for the Department of Fixed Prosthodontics at Osaka Dental University. He is a member of the International Association for Dental Research and the Japan Society of Medical Electronics and Biological Engineering. He is currently 
interested in dynamic analysis of tooth supporting structures.

Sheng-gen Shi received his M.D. and Ph.D degrees in at Stomatology from College of Stomatology, Fourth Military Medical University, China in 1988 and 1992, respectively. $\mathrm{He}$ is an associate professor in the Department of Prosthodontics, College of Stomatology, Fourth Military Medical University, China and a visiting research scientist in the Department of Fixed Prosthodontics, Osaka Dental University, Japan. He is interested in researches related to tooth mobility.

Takayoshi Kawazoe received his D.D.S. degree from Osaka

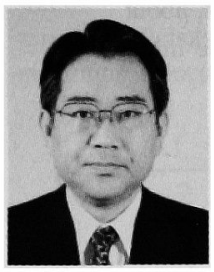

Dental University, Osaka, Japan in 1966 and $\mathrm{Ph} . \mathrm{D}$ there in 1970 . He is presently a professor in the Department of Fixed Prosthodontics at Osaka Dental University. He is a member of the International Association for Dental Research and the Japan Society of Medical Electronics and Biological Engineering. He is currently interested in the diagnosis of occlusion and temporomandibular joint disorders. 